\title{
Figural aftereffects, illusions and the dimension of field dependence ${ }^{1}$
}

\author{
ALEXANDER W. PRESSEY AND GOLDA KOFFMAN ${ }^{2}$
} UNIVERSITY OF MANITOBA

Three groups of Ss consisting of low, intermediate, and high susceptibility to figural aftereffects were tested on the embedded-figures test, the rod-and-frame test, and the Poggendorff illusion. The results showed that, contrary to recent findings by Immergluck, there was no significant relationship between figural aftereffects and measures of field dependence. However, in men there was an inverse relationship between the magnitude of the Poggendorff illusion and figural aftereffects.

In recent studies, Immergluck (1966a, 1966b) has presented evidence that a relationship exists between field dependence and susceptibility to figural aftereffects (FAEs). Field independent Ss give more reports of FAEs than do field dependent Ss. However, Immergluck's results are suspect because of a major weakness in his design, viz., a failure to control for the sex of the $S$. It is well known that women tend to be more field dependent than men (Witkin, et al, 1962), and it has also been shown that women tend to be less susceptible than men to visual FAEs (Koffman \& Pressey, 1967; Paul, 1965; Wertheimer, 1954). Since Immergluck used extreme groups to establish his field dependent and independent groups, It is probable that the field dependent group contained more women and the field independent group more men. In such a case a relationship would be found between FAEs and field dependence, but this relationship could be due to sex rather than to field dependence.

Another drawback in Immergluck's studies is the use of only one test, the rod-and-frame test (RFT), of field dependence and one measure of a FAE. Thus, if the obtained relationship between field dependence and FAE is not due to an artifact of sex, it may be questioned to what degree the relationship is representative of results that would obtain when different measures of field dependence and FAEs are used.

The present study was an attempt to replicate Immergluck's findings under conditions in which sex of $S$ was controlled and more than one measure of field dependence and FAEs were employed.

Method

A group test of visual FAEs was administered to 77 men and 58 women enrolled in an introductory psychology course at the University of Manitoba. A detailed description of the method and procedure for measuring FAEs has been provided elsewhere (Koffman \& Pressey, 1967). Briefly, there were five different patterns for inducing and testing for a FAE. Each pattern consisted of an inspection-figure and a test-figure. For example, one inspection-figure consisted of two unequal circles whose centers were 1 in. to the left and right of a fixation point. The test-figure consisted of two equal circles whose centers were 1 in. to the left and right of the fixation point. After fixating on the inspection-figure for $45 \mathrm{sec}$, $S$ switched his gaze to the test-figure and was asked to report which of the following occurred: right circle larger, left circle larger, no apparent difference. In addition, he was asked to rate the vividness of his experience on a 5-point scale. Two scores were obtained for each $S$. One score was the number of reports of distortion in the expected direction and the second score was the sum of the ratings of vividness on all five tests.

Of the original group, 27 men and 27 women were selected for further testing. These were grouped into three categories consisting of high, intermediate, and low susceptibility to FAEs. The high group contained Ss who reported 4 or 5 FAEs; the intermediate group reported 3 FAEs and the low group reported 0,1 , or 2 FAEs. Within each group men and women were matched on the basis of number of reports of FAEs and vividness of their experience.

The Ss were tested on the embedded-figures test (EFT), the RFT, and the Poggendorff illusion in that order. The illusion was included because it has been found to correlate substantially with both the EFT and the RFT (Pressey, 1967) and because Immergluck (1966c) has reported a significant relationship between geometrical illusions and FAEs. The Ss were tested in a random order by an $\mathrm{E}$ who did not know to which category the $\mathrm{S}$ belonged.

For the EFT, the first 12 figures and methods of administration and scoring described by Witkin (1950) were used. The RFT was Model V-1260M2 designed by the Polymetric Company to conform to the test used in Witkin's original studies. Each $S$ was seated in an upright position at a distance of $12 \mathrm{ft}$ in front of the apparatus. Four trials were given. Prior to each trial the frame and rod were tilted $26 \mathrm{deg}$ either to the left or right. The order of tilt for the frame was LRRL and for the rod it was LLRR. The average error (in deg) from the objective vertical was taken as S's score.

The Poggendorff fllusion consisted of two horizontal lines which interrupted a line tilted 35 deg from the horizontal and to S's right. Each horizontal line was 4 In. long, and each portion of the diagonal line was $1 \mathrm{in.}$ long. The distance between the horizontal lines was .75 in. The upper portion of the diagonal line was 
Table 1. Mean Scores on Three Tests as a Function of Susceptibility to Visual Figural Aftereffects

\begin{tabular}{llccc} 
& & \multicolumn{3}{c}{ Susceptibility to Figural Aftereffects } \\
\cline { 3 - 5 } Tests & Low & Medium & High \\
\hline \multirow{2}{*}{ EFT (sec) } & Men & 29.7 & 56.2 & 45.6 \\
& Women & 31.1 & 41.0 & 35.6 \\
\multirow{2}{*}{ RFT (deg) } & Men & 3.19 & 3.82 & 4.01 \\
& Women & 3.99 & 2.42 & 2.51 \\
Illusion & Men & .243 & .126 & .130 \\
(in.) & Women & .154 & .192 & .131 \\
\hline
\end{tabular}

variable and could be adjusted by means of a knob located at the upper-right corner of a metal frame on which the illusion was mounted. A chin rest was placed in front of the apparatus so that the distance between S's eyes and the target was 12 in. A total of four trials, alternating in direction of adjustment, was given. The S's score was the average deviation from the point of objective alignment measured to within .001 in.

\section{Results and Discussion}

The mean score on each test as a function of susceptibility to FAEs is presented in Table 1. An analysis of variance in which the two main effects were sex of $S$ and susceptibility to FAEs was carried out on each of the three tests. None of the $F$ ratios was significant at the .05 level on the EFT and the RFT. However, on the Poggendorff illusion the $F$ ratio for the effect of FAEs was 3.65 ( df $=2 / 48$, $p<.05)$ and for the sex by FAE interaction the $F$ value was $4.76(\mathrm{df}=2 / 48, \mathrm{p}<.05)$. The best interpretation of this interaction, according to the data, is that men who are highly susceptible to FAEs have markedly smaller illusions than men who are less susceptible to FAEs. Differences in FAEs in women, however, did not relate to performance on the Poggendorff illusion.

In conclusion, the present study shows that, when sex of $\mathrm{S}$ is controlled, there is no relationship between visual FAEs and field dependence. However, Immergluck's finding that Ss who are more susceptible to FAEs were less susceptible to geometrical illusions is upheld-at least for men.

\section{References}

IMMERGLUCK, L. Visual figural aftereffects and field dependence. Psychon. Sci, 1966a, 4, 219-220.

IMMERGLUCK, L. Figural after-effects, rate of "figure-ground" reversal, and field dependence. Psychon. Sci., 1966b, 6, 45-46.

IMMERGLUCK, L. Resistance to an optical illusion, figural after-effects, and field dependence. Psychon. Sci., 1966c, 6, 281-282.

KOFFMAN, G., \& PRESSEY, A. W. Sex differences on a group test of figural aftereffects. Psychon. Sci., 1967, 8, 511-512.

PAUL, S. K. A note on sex differences in figural aftereffects. Psychological Studies, 1965, 10, 143-144.

PRESSEY, A. W. Field dependence and susceptibility to the Poggendorff illusion. Percept. mot. Skills, 1967, 24, 309-310.

WERTHEIMER, M. The differential satiability of schizophrenic and normal subjects: A test of a deduction from the theory of figural after-effects. J. gen. Psychol., 1954, 51, 291-299.

WITKIN, H. A. Individual differences in ease of perception of embedded figures. J. Pers., 1950, 19, 1-15.

WITKIN, H. A., DYK, R. B., FATERSON, H. F., GOODENOUGH, D. R., \& KARP, S. A. Psychological differentiation. New York: Wiley, 1962.

Notes

1. This research was supported in part by the National Research Council of Canada (APA-177).

2. Now at the University of Alberta, Edmonton. 